\title{
A Soluble Guanylate Cyclase Activator Inhibits the Progression of Diabetic Nephropathy in the ZSF1 Rat
}

\author{
Carine M. Boustany-Kari, Paul C. Harrison, Hongxing Chen, Kathleen A. Lincoln, \\ Hu Sheng Qian, Holly Clifford, Hong Wang, Xiaomei Zhang, Kristina Gueneva-Boucheva, \\ Todd Bosanac, Diane Wong, Ryan M. Fryer, Jeremy G. Richman, Chris Sarko,
} and Steven S. Pullen

Departments of Cardiometabolic Diseases Research (C.M.B.-K., P.C.H., H.C., K.A.L., H.S.Q., H.C., H.W., X.Z., R.M.F., J.G.R., S.S.P.) and Small Molecule Discovery Research (K.G.-B., T.B., D.W., C.S.), Boehringer Ingelheim Pharmaceuticals, Ridgefield, Connecticut

Received November 12, 2015; accepted December 30, 2015

\begin{abstract}
Therapies that restore renal cGMP levels are hypothesized to slow the progression of diabetic nephropathy. We investigated the effect of BI 703704, a soluble guanylate cyclase (sGC) activator, on disease progression in obese ZSF1 rats. BI 703704 was administered at doses of $0.3,1,3$, and $10 \mathrm{mg} / \mathrm{kg} / \mathrm{d}$ to male ZSF1 rats for 15 weeks, during which mean arterial pressure (MAP), heart rate $(\mathrm{HR})$, and urinary protein excretion (UPE) were determined. Histologic assessment of glomerular and interstitial lesions was also performed. Renal cGMP levels were quantified as an indicator of target modulation. BI 703704 resulted in sGC activation, as evidenced by dose-dependent increases in renal cGMP levels.
\end{abstract}

After 15 weeks of treatment, sGC activation resulted in dosedependent decreases in UPE (from $463 \pm 58 \mathrm{mg} / \mathrm{d}$ in vehicle controls to $328 \pm 55,348 \pm 23,283 \pm 45$, and $108 \pm 23 \mathrm{mg} / \mathrm{d}$ in $\mathrm{BI} 703704$-treated rats at $0.3,1,3$, and $10 \mathrm{mg} / \mathrm{kg}$, respectively). These effects were accompanied by a significant reduction in the incidence of glomerulosclerosis and interstitial lesions. Decreases in MAP and increases in HR were only observed at the high dose of BI 703704. These results are the first demonstration of renal protection with SGC activation in a nephropathy model induced by type 2 diabetes. Importantly, beneficial effects were observed at doses that did not significantly alter MAP and HR.

\section{Introduction}

Diabetic nephropathy is the most common cause of end-stage renal disease (ESRD) in Western societies and is characterized by a progressive decline in renal function accompanied by mesangial expansion, glomerular basement membrane thickening, and tubulointerstitial damage (Tervaert et al., 2010; Saran et al., 2015). Diabetic nephropathy patients receiving current standard of care treatment, namely angiotensinconverting enzyme (ACE) inhibitors and angiotensin receptor blockers (ARB), continue to progress to ESRD, indicating a clear unmet medical need for new therapies that will slow or arrest renal function decline in these patients (Saran et al., 2015).

Endothelial dysfunction has been linked in humans and preclinically with the progression of diabetic nephropathy. Increased plasma levels of endothelial dysfunction markers, namely von Willebrand factor, soluble vascular cell adhesion molecule-1, and soluble intercellular cell adhesion molecule-1, have been associated with disease progression in diabetic nephropathy patients (Persson et al., 2008). Furthermore, asymmetric dimethyl arginine, an endogenous inhibitor of

dx.doi.org/10.1124/jpet.115.230706. endothelial nitric oxide synthase (eNOS), is associated with the development and progression of diabetic nephropathy in patients (Lajer et al., 2008; Hanai et al., 2009; Shibata et al., 2009). Consistent with these studies, eNOS polymorphisms resulting in reduced enzyme expression or function have been associated with diabetic nephropathy (Dellamea et al., 2014). In an analysis of preclinical models of disease, it was demonstrated that deficiency of eNOS in $\mathrm{db} / \mathrm{db}$ diabetic mice produced a renal gene expression profile that more closely resembled human diabetic nephropathy, compared with $\mathrm{db} / \mathrm{db}$ mice with intact eNOS (Hodgin et al., 2013). Taken together, these studies highlight a role for impaired nitric oxide (NO) production/ signaling in the progression of diabetic nephropathy.

A key messenger for NO signaling is cGMP, the product of GTP catalysis by soluble guanylate cyclase (sGC) (Lewicki et al., 1982). Under conditions of oxidative stress such as those found in diabetes, the heme prosthetic group of sGC becomes oxidized, rendering it nonresponsive to NO (Stasch et al., 2006). This finding has led to the generation of pharmacological compounds aimed at restoring cGMP levels, thereby repairing endothelial dysfunction. As such, a class of synthetic compounds termed sGC activators has been demonstrated to bypass NO signaling impairment by displacing the defective

ABBREVIATIONS: ACE, angiotensin-converting enzyme; ARB, angiotensin receptor blocker; bpm, beats per minute; eNOS, endothelial nitric oxide synthase; ESRD, end-stage renal disease; HR, heart rate; KIM-1, kidney injury molecule 1; MAP, mean arterial pressure; NO, nitric oxide; sGC, soluble guanylate cyclase; $\alpha$-SMA, $\alpha$ smooth muscle actin; T2DM, type 2 diabetes mellitus; UPE, urinary protein excretion. 
heme prosthetic group, thereby stabilizing the enzyme in an activated conformation and restoring cGMP generation (Stasch et al., 2002). Another class of compounds termed sGC stimulators is also able to increase cGMP production by sGC, but not when the heme prosthetic group has been oxidized (Stasch et al., 2001).

Given that diabetic nephropathy is associated with endothelial dysfunction and increased oxidative stress, we sought to investigate the effect of $\mathrm{SGC}$ activation on the progression of the disease in the ZSF1 rat. The ZSF1 $\left(L e p^{f a} / L e p r^{c p}\right)$ obese rat, a hybrid cross between ZDFo $\left(L e e^{f a}\right)$ and $\operatorname{SHHFơ~}\left(L e p r^{c p}\right)$, was selected for these studies, as it exhibits many of the traits of human diabetic nephropathy, including proteinuria, renal lesions, hyperglycemia, dyslipidemia, mild hypertension, oxidative stress, and obesity (Tofovic and Jackson, 2003; Boerrigter and Burnett, 2007; Prabhakar et al., 2007). Our findings indicate a pronounced and dose-dependent effect of BI 703704 (Fig. 1; Berry et al., 2013), a sGC activator, to reduce progression of renal damage in the ZSF1 rat, and highlight the potential of sGC activation as an effective therapy for diabetic nephropathy.

\section{Materials and Methods}

All procedures were conducted in accordance with the Guide for the Care and Use of Laboratory Animals as adopted and promulgated by the National Institutes of Health, and under an Institutional Animal Care and Use Committee-approved study protocol at Boehringer Ingelheim Pharmaceuticals (Ridgefield, CT).

\section{Animals}

Male ZSF1 obese rats were obtained from Charles River Laboratories (Kingston, NY) at 10-11 weeks of age following surgical implantation of telemetry transmitters (Data Sciences International; PA11TA-C40, New Brighton, MN) in the abdominal artery to allow for continuous monitoring of mean arterial pressure (MAP) and heart rate $(\mathrm{HR})$. Rats were acclimated to metabolism cages (Laboratory Products, Seaford, DE) for 1 week prior to baseline collections, and remained in these cages throughout the study to allow for weekly collection of urine. Baseline measurements were collected 1 week prior to start of treatment.

Experimental Design. Fifty-eight male ZSF1 rats (12-13 weeks of age) were randomly assigned based on their average baseline urinary protein excretion (UPE), MAP, and body weight to receive either enalapril ( $3 \mathrm{mg} / \mathrm{kg} / \mathrm{d}$ in the drinking water, $n=10 /$ group) or one of four doses of BI 703704 (0.3, 1, 3, or $10 \mathrm{mg} / \mathrm{kg} / \mathrm{d}, n=9-10 /$ group) incorporated into the diet (Purina Formulab 5008; WF Fisher \& Son, Somerville, NJ). Dietary concentrations of BI 703704 were estimated

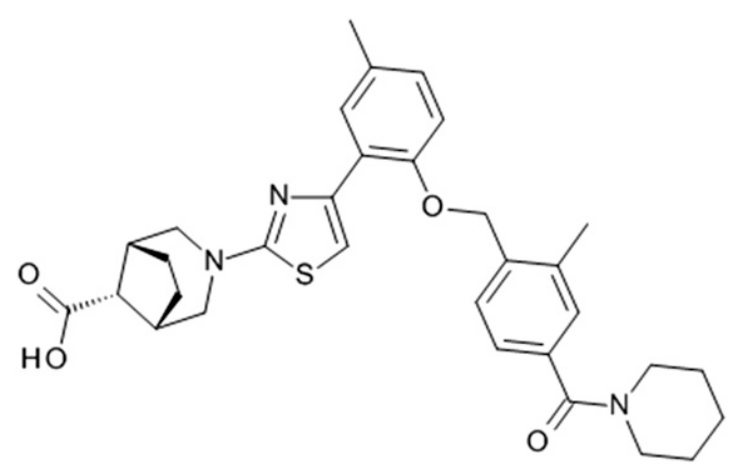

Fig. 1. Structure of BI 703704. based on historical data of daily food intake ( $33 \mathrm{~g} / \mathrm{d})$ and body weight of ZSF1 rats, and were adjusted every 5 weeks. Rats were subsequently monitored for 15 weeks, during which body weight was recorded once per week, and food intake and water intake thrice per week. Urine was collected chilled once weekly over a 24 -hour period for measurement of protein contents. Blood samples were collected at baseline, following 5 and 10 weeks of treatment, and at study termination for pharmacokinetic, clinical chemistry, plasma glucose, and HbA1c measurements. Hemodynamic parameters were recorded intermittently (1 minute of continuous data every 10 minutes), and weekly 24-hour averages were calculated. At study end, rats were sacrificed by exsanguination through cardiac puncture under isoflurane anesthesia, and kidneys were collected for histologic analysis and determination of drug concentrations and cGMP levels.

Biochemical and Pharmacokinetic Measurements. Following centrifugation (930 rcf for 10 minutes), urine samples were diluted in $0.1 \mathrm{~N} \mathrm{NaOH}$ and protein concentration was determined using Coomassie Plus Reagent (Thermo Fisher, Waltham, MA; \#23238).

The quantitation of enalaprilat and BI 703704 in sodium citrate acidified with citric acid plasma and kidney cortex was performed using liquid chromatography and mass spectrometry. An API 5000 triple-quadruple mass spectrometer with Turbo V Ion Source (Applied Biosytems, Toronto, Canada), set to electrospray positive ionization mode, and Analyst 1.4.2 operating software were used. Ion transitions used for quantification were as follows: enalaprilat $(349.0-206.0 \mathrm{~m} / \mathrm{z}$, CE 25, DP 80V) and BI 703704 (560.2-216.2 m/z, CE 40, DP 80V). The liquid chromatography system was an Agilent 1200 Series pump and column oven (Fullerton, CA) with a LEAP Technologies HTS-PAL Autosampler (Carrboro, NC). The analytical column was a Phenomenex Synergi Polar RP, $2.1 \times 50 \mathrm{~mm}, 4 \mu \mathrm{m}$ (Torrance, CA), and the mobile phase consisted of $10 \mathrm{mM}$ ammonium acetate with $0.1 \%$ formic acid in water (A) and acetonitrile (B). Gradient was maintained at $5 \% \mathrm{~B}$ for 0.1 minute, increased to $95 \% \mathrm{~B}$ in 1.8 minutes, held at $95 \% \mathrm{~B}$ for 0.5 minute, and decreased to $5 \% \mathrm{~B}$ for 0.2 minute and held at $5 \% \mathrm{~B}$ for a total time of 3 minutes at a flow rate of $0.5 \mathrm{ml} / \mathrm{min}$. The total run time per injection was 3 minutes.

Sample preparation. Enalaprilat and BI 703704 standard stock solutions containing $1 \mathrm{mg} / \mathrm{mL}$ in methanol were serially diluted in rat plasma to prepare an 8-point standard curve ranging from 1 to $5000 \mathrm{ng} / \mathrm{mL}$. A total of $20 \mu \mathrm{L}$ plasma or homogenized kidney samples, calibration standards, and blank plasma samples was deproteinized by precipitation with $180 \mu \mathrm{L}$ Internal Standard (100 ng/mL; proprietary small molecule) diluted in acetonitrile:water (85:15). Samples were mixed for 1 minute, filtered through AcroPrep multiwell filter plates (Pall, Ann Arbor, MI) using Sciclone ALH 3000 Workstation (Caliper Life Sciences, Hopkinton, MA), and transferred into 96-well injection plate. A total of $5 \mu \mathrm{L}$ solution was then injected into the liquid chromatography/mass spectrometry/mass spectrometry system for analysis.

cGMP levels were determined in frozen kidney cortex sections. Tissues were pulverized using a ceramic mortar and transferred into prechilled tubes containing matrix-homogenizing beads (MP Biomedicals Life Sciences, Newport Beach, CA) for further disruption. Following acidification and acetylation, cGMP levels were determined using a cGMP ELISA kit (EnZO Life Sciences Direct, Farmingdale, NY).

Protein levels were measured in urine collected weekly over a 24-hour period. Protein contents were quantified utilizing a Bradford assay (Coomassie Plus; Thermo Fisher Scientific, Waltham, MA). HbA1c was measured by high-pressure liquid chromatography (DTI Laboratories, Thomasville, GA). Fasting plasma glucose was measured utilizing a glucose meter (Bayer Breeze2; Bayer HealthCare LLC, Mishawaka, IN).

Tissue Collection and Processing. For histologic assessment, kidneys were removed and weighed, and a mid-organ transverse section of the left kidney was collected and immediately fixed by immersion in $10 \%$ phosphate-buffered formalin for 48 hours. Subsequently, formalin-fixed tissues were washed in phosphate buffer, dehydrated through a graded series of ethanol and xylene, embedded in paraffin, and sectioned at $4 \mu \mathrm{m}$. 
General Assessment of Renal Histopathology. Kidney tissue sections $(4 \mu \mathrm{m})$ were stained with periodic acid methenamine silver for the general assessment of incidence of glomerulosclerosis and $\mathrm{H} \& \mathrm{E}$ for the general assessment of total number of foci of interstitial lesions. For the glomerular lesions, the number of glomeruli showing mild to severe glomerulosclerosis was counted in each kidney section and expressed as a percentage of the total glomerular population. Tubulointerstitial lesions were assessed as the number of foci showing obvious renal damage, which included marked hypercellularity (inflammation or resident myofibroblast increases), fibrosis, tubular hyperplasia, casts, or necrosis. Both assessments were performed under blinded conditions at $10 \times$ magnification.

Immunohistochemistry and Image Analysis. Four micrometer kidney sections were air dried overnight at $37^{\circ} \mathrm{C}$, dewaxed, and rehydrated in graded ethanol to phosphate-buffered saline. Endogenous peroxidase activity was blocked by ImmPRESS (Vector Laboratories, Burlingame, CA) for 20 minutes at ambient temperature. Sections were then washed and incubated with the primary antibody for $\alpha$-smooth muscle actin ( $\alpha$-SMA; A5228; Sigma-Aldrich, St. Louis, MO) at a dilution of 1:3000 and kidney injury molecule-1 (KIM-1; AF3689; R\&D Systems, Minneapolis, MN) at a dilution of 1:200 and were subsequently incubated with the respective secondary antibodies (ImmPress Reagent Kit; Vector Laboratories, Burlingame, CA) for 30 minutes in a hydration chamber. Immunoperoxidase detection was performed using the avidin-biotin complex method (Vector Laboratories) using 3,3'-diaminobenzidine tetrahydrochloride as substrate.

Quantitative image analysis of $\alpha$-SMA and KIM- 1 staining was performed under blinded conditions. Ten random digital images were captured at $10 \times$ objective magnification, with each field representing approximately $0.6 \mathrm{~mm}^{2}$ for a total area of $6.0 \mathrm{~mm}^{2}$ being assessed. The percentage of positive staining area per field in the cortex region was measured using computer-assisted image analysis with Image-Pro imaging software.

Statistical Analysis. Data collected repetitively over time were analyzed using a two-way analysis of variance with repeated measures and a Bonferroni post hoc analysis. Data obtained through single measurements (cGMP, histologic endpoints) were analyzed using a oneway analysis of variance with Tukey's post hoc analysis. Significance was achieved at $P<0.05$. Statistical analysis was performed using GraphPad Prism Version 6.01 (GraphPad Software, La Jolla, CA).

Pharmacokinetics/Pharmacodynamics Modeling. Renal cGMP changes were correlated with BI 703704 individual mean plasma concentrations using an $\mathrm{E}_{\max }$ model. Additionally, changes from baseline in UPE were correlated with BI 703704 individual mean plasma concentrations using an $\mathrm{I}_{\max }$ model. All analyses were performed using Phoenix WinNonlin Version 6.1 (Pharsight, Cary, NC).

\section{Results}

Pharmacokinetics of BI 703704 in Plasma and Kidney. Administration of BI 703704 at $0.3,1,3$, and $10 \mathrm{mg} / \mathrm{kg} / \mathrm{d}$ yielded plasma concentrations that were dose-dependent throughout the study (Table 1). Following 15 weeks of treatment, BI 703704 renal concentrations were also dose-dependent, demonstrating presence of compound in the target organ (Table 1). Plasma concentrations of enalaprilat, the active metabolite of enalapril, were consistent across the study (Table 1).

Effect of sGC Activation on cGMP Content in the Kidney. Renal content of cGMP, a biomarker of sGC activation, was determined in the kidney cortex at study termination. BI 703704 treatment resulted in a dose-dependent increase in renal cGMP content $(16.75 \pm 1.86,23.85 \pm 2.65$, $24.84 \pm 2.08,28.02 \pm 2.55 \mathrm{pmol} / \mathrm{g}$ kidney tissue at doses of 0.3 , 1,3 , and $10 \mathrm{mg} / \mathrm{kg}$, respectively; $P<0.05$ versus vehicle), indicating that the compound had resulted in an activation of sGC in vivo within the target organ. At the dose of $10 \mathrm{mg} / \mathrm{kg}$, cGMP content was significantly greater than seen in the vehicle $(18.01 \pm 1.55 \mathrm{pmol} / \mathrm{g}$ tissue $)$ and enalapril (14.98 \pm $1.17 \mathrm{pmol} / \mathrm{g}$ tissue) groups. To derive BI 703704 in vivo potency in activating sGC, renal cGMP content was associated with plasma and renal drug concentrations utilizing an $\mathrm{E}_{\max }$ model. The plasma concentration of BI 703704 was correlated with renal cGMP content (Fig. 2), and the plasma concentration necessary to elicit a $50 \%$ increase in renal cGMP content $\left(\mathrm{EC}_{50}\right)$ was estimated to be $420 \mathrm{nM}(108 \% \mathrm{CV})$. Similarly, BI 703704 renal concentrations were correlated with renal cGMP content, and the $\mathrm{EC}_{50}$ was estimated to be $105 \mathrm{nM}$ (93\% CV). These results demonstrate that increasing concentrations of BI 703704 in the systemic circulation as well as in the target organ lead to production of renal cGMP, thereby validating that BI 730704 effectively modulates sGC in a concentrationdependent manner.

Effect of sGC Activation on Metabolic Measurements. At baseline, there was no significant difference in body weight, food intake, or water intake between study groups (Table 2). Following BI 703704 administration, there was a significant, dose-dependent increase in body weight gain versus vehicle, despite a decrease in food and water intake at the doses of 3 and $10 \mathrm{mg} / \mathrm{kg}$. In contrast, enalapril did not significantly affect body weight or food and water intake compared with vehicle. Concomitantly with the reductions in food intake, $\mathrm{HbA1c}$ levels were significantly decreased by the end of the study at the doses of 3 and $10 \mathrm{mg} / \mathrm{kg}$ BI 703704 (change from baseline in HbA1c \%: vehicle, $1.1 \pm 0.2$; enalapril, $1.1 \pm 0.2 ; \mathrm{BI} 7037040.3 \mathrm{mg} / \mathrm{kg}, 0.5 \pm 0.2 ; 1 \mathrm{mg} / \mathrm{kg}, 0.5$ $\pm 0.2 ; 3 \mathrm{mg} / \mathrm{kg}, 0.3 \pm 0.1^{*} ; 10 \mathrm{mg} / \mathrm{kg},-0.9 \pm 0.1^{* \#} ; * P<0.05$ versus vehicle, ${ }^{\#} P<0.05$ versus enalapril). Nonfasting plasma glucose was not significantly different between groups by week 15 (change from baseline in plasma glucose: vehicle, $106.8 \pm 52.9$; enalapril, $119.9 \pm 34.6$; BI $7037040.3 \mathrm{mg} / \mathrm{kg}$,

TABLE 1

Mean plasma and intrarenal concentrations of BI 703704 and enalaprilat

\begin{tabular}{lccccc}
\hline & & \multicolumn{3}{c}{ Mean Concentration } \\
\cline { 3 - 5 } Compound & Dose $(\mathrm{mg} / \mathrm{kg} / \mathrm{day})$ & \multicolumn{3}{c}{ Plasma } & Kidney \\
\cline { 3 - 5 } & & Week 5 & Week 10 & Week 15 & Week 15 \\
\cline { 3 - 5 } & & $101 \pm 9$ & $78 \pm 4$ & $94 \pm 19$ & $25 \pm 2$ \\
BI 703704 & 0.3 & $524 \pm 100$ & $319 \pm 22$ & $496 \pm 114$ & $83 \pm 5$ \\
& 1 & $1129 \pm 80$ & $1082 \pm 79$ & $1358 \pm 247$ & $243 \pm 15$ \\
& 3 & $3455 \pm 455$ & $2815 \pm 165$ & $3175 \pm 130$ & $1509 \pm 114$ \\
Enalaprilat & 10 & $120 \pm 21$ & $141 \pm 21$ & $212 \pm 23$ & $124 \pm 13$ \\
\hline
\end{tabular}

Plasma levels reported as mean \pm S.E.M. in nM for BI 703704 and enalaprilat, the active metabolite of enalapril, at weeks 5, 10, and 15 of the study. Kidney concentrations reported in nM for BI 703704 and enalaprilat at week 15. 


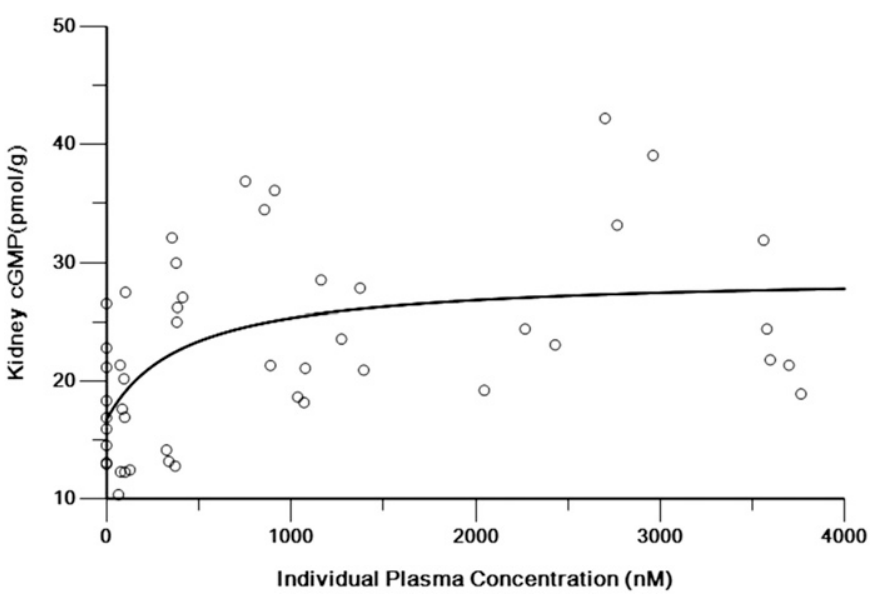

Fig. 2. Correlation of BI 703704 plasma concentrations with kidney cGMP contents. Using a simple $\mathrm{E}_{\max }$ model, individual mean plasma concentrations (nM; mean of three time points) of BI 703704 were associated with respective kidney cGMP contents (pmol/g tissue) measured after 15 weeks of treatment. Increasing kidney cGMP contents correlated with increasing BI 703704 concentrations, and an $\mathrm{EC}_{50}$ of $420 \mathrm{nM}(108 \% \mathrm{CV})$ was calculated as the BI 703704 plasma concentration associated with a $50 \%$ increase in renal cGMP.

$66.11 \pm 41.4 ; 1 \mathrm{mg} / \mathrm{kg},-53.22 \pm 36.6 ; 3 \mathrm{mg} / \mathrm{kg},-17.11 \pm$ $35.35 ; 10 \mathrm{mg} / \mathrm{kg}, 10.78 \pm 29.3 \mathrm{mg} / \mathrm{dl} ; P>0.05)$.

Effect of sGC Activation on Hemodynamic Measurements. At baseline, there was no significant difference in MAP or HR between study groups [MAP: $119 \pm 3,117 \pm 1,119 \pm 1$, $119 \pm 2,117 \pm 2$, and $118 \pm 1 \mathrm{mmHg}$ and HR: $316 \pm 5,305 \pm 10$, $320 \pm 4,323 \pm 3,318 \pm 5$, and $315 \pm 4$ beats per minute $(\mathrm{bpm})$ for vehicle, enalapril, and BI 703704 at $0.3,1,3$, and $10 \mathrm{mg} / \mathrm{kg}$, respectively; $P>0.05]$. Administration of $\mathrm{BI} 703704$, at the dose of $10 \mathrm{mg} / \mathrm{kg}$, resulted in a significant decrease in MAP starting from week 2 onward (Fig. 3). The doses of $0.3,1$, and $3 \mathrm{mg} / \mathrm{kg}$ did not significantly affect MAP compared with vehicle. In comparison, enalapril, tested at $3 \mathrm{mg} / \mathrm{kg}$, resulted in a significant reduction in MAP versus vehicle from week 11 onward.

HR decreased over time in all study groups, including vehicle. At the highest dose tested, BI 703704 resulted in a significant increase in HR versus vehicle and enalapril (week $15 \mathrm{HR}$ : vehicle, $265 \pm 5 \mathrm{bpm}$ and BI 703704 at $10 \mathrm{mg} / \mathrm{kg}, 297 \pm 5 \mathrm{bpm}$; Fig. 4). The doses of $0.3,1$, and $3 \mathrm{mg} / \mathrm{kg}$ did not elicit a significant effect on HR. Similarly, enalapril did not significantly alter HR.

Effect of sGC Activation on Renal Parameters. At baseline there was no significant difference in proteinuria between study groups. BI 703704 treatment resulted in
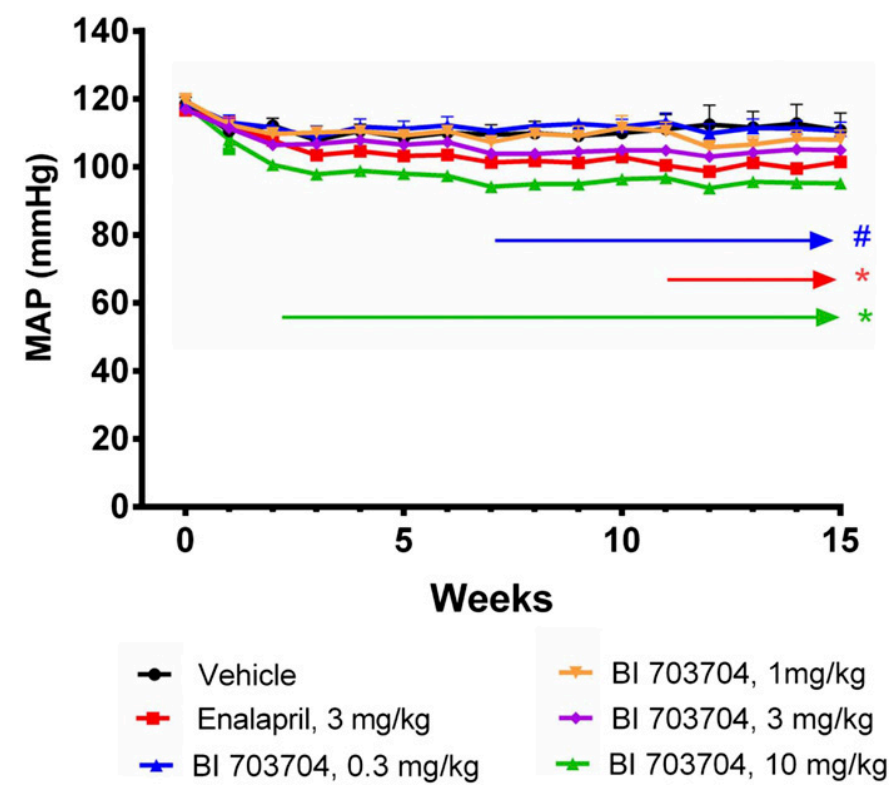

Fig. 3. Effect of sGC activation on weekly MAP. Beginning at week 2, BI 703704 at $10 \mathrm{mg} / \mathrm{kg}$ resulted in a significant reduction in MAP compared with vehicle. There were no relevant significant effects of BI 703704 at $0.3,1$, and $3 \mathrm{mg} / \mathrm{kg}$ on MAP. Enalapril at $3 \mathrm{mg} / \mathrm{kg}$ showed a significant reduction in MAP from week 11 onward. ${ }^{* \#}$ Indicate statistical significance compared with vehicle and enalapril $(3 \mathrm{mg} / \mathrm{kg})$, respectively $(P<0.05$, two-way analysis of variance with repeated measures and Bonferrroni post hoc analysis)

dose-dependent decreases in proteinuria (week 15 UPE: vehicle, $463 \pm 58 \mathrm{mg} / \mathrm{d}$, and BI 703704, $328 \pm 55,348 \pm 23$, $283 \pm 45$, and $108 \pm 23 \mathrm{mg} / \mathrm{d}$ at $0.3,1,3$, and $10 \mathrm{mg} / \mathrm{kg}$, respectively; Fig. 5). The effects of BI 703704 were significantly different from vehicle at doses of 1,3 , and $10 \mathrm{mg} / \mathrm{kg} / \mathrm{d}$, and from enalapril at doses of 3 and $10 \mathrm{mg} / \mathrm{kg} / \mathrm{d}$. To determine BI 703704 in vivo potency in reducing UPE, plasma concentrations of BI 703704 were correlated with UPE (as a change from baseline) utilizing an $\mathrm{I}_{\max }$ model (Fig. 6). The plasma concentration of BI 703704 necessary to elicit a 50\% decrease in UPE $\left(\mathrm{IC}_{50}\right)$ was estimated to be $788 \mathrm{nM}(36 \% \mathrm{CV})$, consistent with its potency in activating renal cGMP production.

Following 15 weeks of treatment with BI 703704, rats were sacrificed and kidney weights were determined. BI 703704 resulted in a dose-dependent reduction in both left and right kidney weights normalized to body weight (average kidney weight/body weight ratio: vehicle, $0.392 \pm 0.019$ and $\mathrm{BI}$ $703704,0.364 \pm 0.023,0.333 \pm 0.014,0.316 \pm 0.017$, and

TABLE 2

Effect of BI 703704 and enalapril on metabolic parameters

\begin{tabular}{|c|c|c|c|c|c|c|}
\hline \multirow{2}{*}{ Treatment } & \multicolumn{2}{|c|}{ Body Weight (g) } & \multicolumn{2}{|c|}{ Food Intake (g) } & \multicolumn{2}{|c|}{ Water Intake (g) } \\
\hline & Baseline (Day 0) & $\begin{array}{c}\text { Weight Gain } \\
\text { (through Week 15) }\end{array}$ & Baseline (Day 0) & $\begin{array}{c}\text { Cumulative } \\
\text { (through Week 15) }\end{array}$ & Baseline (Day 0) & $\begin{array}{c}\text { Cumulative } \\
\text { (through Week 15) }\end{array}$ \\
\hline Vehicle & $501 \pm 7$ & $211 \pm 10$ & $34 \pm 1$ & $3527 \pm 148$ & $55 \pm 8$ & $8452 \pm 916$ \\
\hline Enalapril, $3 \mathrm{mg} / \mathrm{kg}$ & $504 \pm 10$ & $178 \pm 9$ & $32 \pm 1$ & $3294 \pm 89$ & $50 \pm 6$ & $7507 \pm 682$ \\
\hline BI $703704,0.3 \mathrm{mg} / \mathrm{kg}$ & $502 \pm 10$ & $220 \pm 13$ & $34 \pm 2$ & $3181 \pm 165$ & $46 \pm 4$ & $7471 \pm 1440$ \\
\hline BI $703704,1 \mathrm{mg} / \mathrm{kg}$ & $507 \pm 11$ & $276 \pm 26^{* \#}$ & $33 \pm 1$ & $3127 \pm 67$ & $53 \pm 5$ & $5745 \pm 315$ \\
\hline BI $703704,3 \mathrm{mg} / \mathrm{kg}$ & $488 \pm 8$ & $273 \pm 9^{* \#}$ & $35 \pm 1$ & $2957 \pm 48^{*}$ & $54 \pm 5$ & $4671 \pm 245^{*}$ \\
\hline BI $703704,10 \mathrm{mg} / \mathrm{kg}$ & $500 \pm 5$ & $313 \pm 8^{* \#}$ & $31 \pm 1$ & $3030 \pm 91^{*}$ & $38 \pm 5$ & $4378 \pm 170^{* \#}$ \\
\hline
\end{tabular}

Metabolic parameters of body weight, food intake, and water intake shown at baseline (day 0) and following 15 weeks of treatment.

${ }^{*}$,\# Significant $P<0.05$ versus vehicle and enalapril, respectively, one-way analysis of variance. 


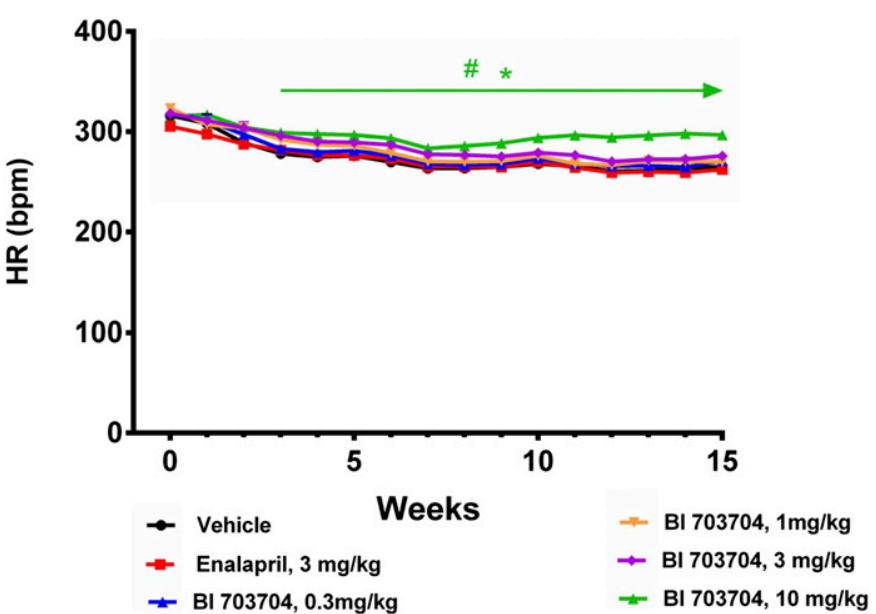

Fig. 4. Effect of sGC activation on weekly mean HR. BI 703704 at $10 \mathrm{mg} / \mathrm{kg}$ resulted in a significant increase in HR beginning on week 4 . There was no significant effect on HR of BI 703704 at $0.3,1$, and $3 \mathrm{mg} / \mathrm{kg}$, or enalapril at $3 \mathrm{mg} / \mathrm{kg}$. ${ }^{* \#}$ Signifies statistical significance compared with vehicle or enalapril, respectively $(P<0.05$, two-way analysis of variance with repeated measures and Bonferrroni post hoc analysis).

$0.258 \pm 0.006$ at $0.3,1,3$, and $10 \mathrm{mg} / \mathrm{kg}$, respectively). Reductions were significantly different from vehicle at the doses of 3 and $10 \mathrm{mg} / \mathrm{kg}$ (reductions of $19 \%$ and $34 \%$ for left kidney, $P<0.05)$. Enalapril $(3 \mathrm{mg} / \mathrm{kg} / \mathrm{d})$ had no effect on kidney to body weight ratio $(0.388 \pm 0.013)$ compared with vehicle control $(0.392 \pm 0.019)$.

Enalapril at the dose tested reduced modestly, but not significantly, the incidence of glomerulosclerosis (20\% decrease versus vehicle; $P>0.05$ ). In comparison, BI 703704 resulted in a significant, dose-dependent decrease in glomerulosclerosis compared with vehicle $(44,48$, and $71 \%$ versus vehicle at the doses of 1,3 , and $10 \mathrm{mg} / \mathrm{kg}$, respectively; Figs. 7 and 8A). Additionally, at the highest dose tested, BI 703704

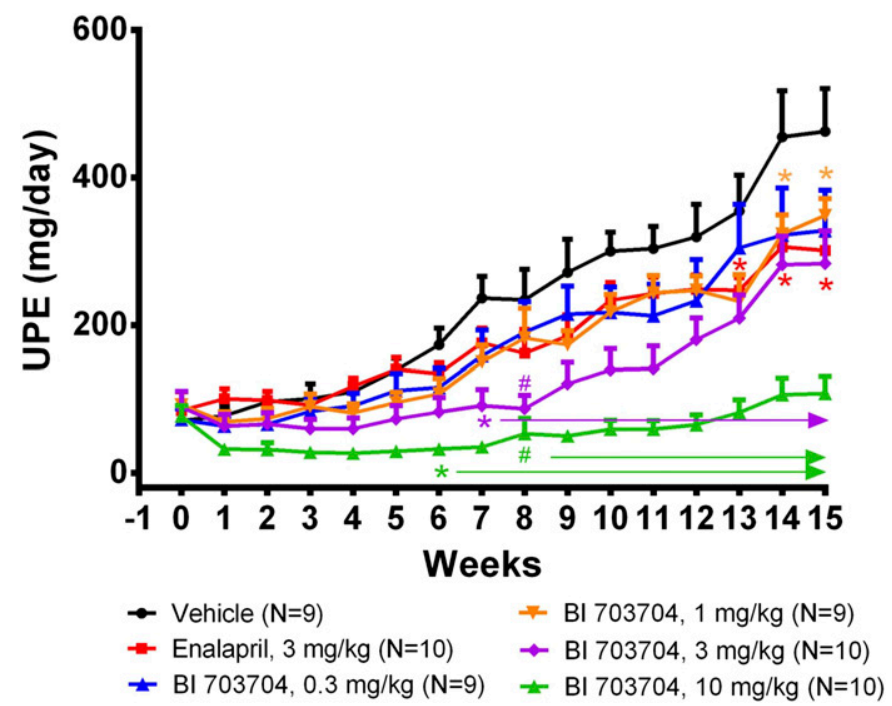

Fig. 5. Effect of sGC activation on weekly UPE. BI 703704 at 1, 3, and 10 $\mathrm{mg} / \mathrm{kg}$ significantly reduced UPE levels from weeks 14,7 , and 6 onward, respectively. Enalapril at $3 \mathrm{mg} / \mathrm{kg}$ significantly reduced UPE levels from week 13 onward. ${ }^{* \#}$ Signifies statistical significance compared with vehicle and enalapril, respectively $(P<0.05$, two-way analysis of variance with repeated measures and Bonferroni post hoc analysis).

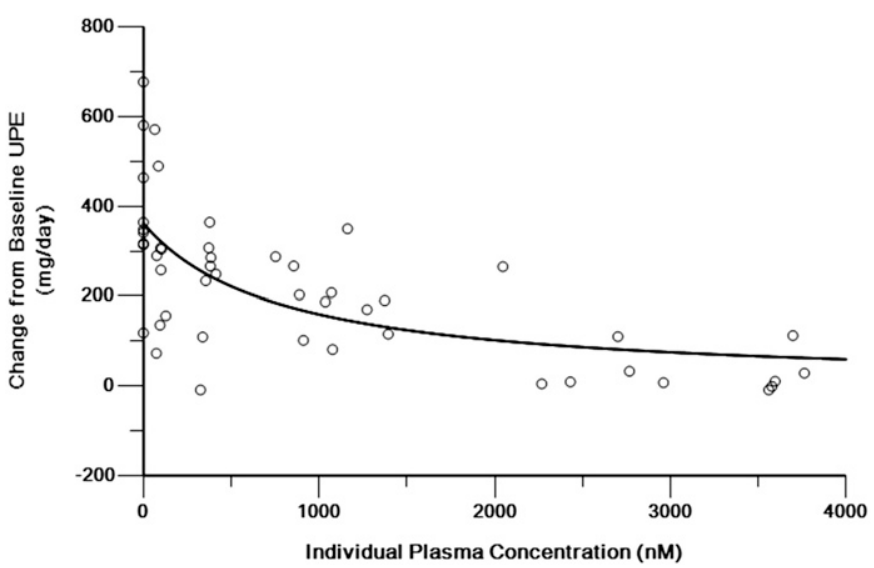

Fig. 6. Correlation of BI 703704 plasma concentrations with UPE. Using a simple Imax model, BI 703704 individual mean plasma concentrations on weeks 5,10 , and 15 were associated with the overall change from baseline UPE levels ( $\mathrm{mg} / \mathrm{d}$ ) for individual animals. Decreasing UPE levels correlated with increasing BI 703704 plasma concentrations, and an $\mathrm{IC}_{50}$ of $788 \mathrm{nM}(36 \% \mathrm{CV})$ was calculated as the BI 703704 plasma concentration associated with a $50 \%$ reduction in UPE.

was significantly different from enalapril. Tubulointerstitial lesions were also dose-dependently decreased by BI 703704 at $0.3,1,3$, and $10 \mathrm{mg} / \mathrm{kg}$ compared with vehicle $(14,21,33$, and $67 \%$, respectively); however, this was only significant in the group receiving BI 703704 at $10 \mathrm{mg} / \mathrm{kg}$ (Fig. 8B). Enalapril reduced tubulointerstitial lesions (24\% versus vehicle) modestly, but not significantly.

Markers of renal damage, including $\alpha$-SMA, a marker of myofibroblast activation, and KIM-1, a marker for renal tubular injury, were quantified in kidney cortex tissue by immunohistochemistry. BI 703704 resulted in a significant reduction in $\alpha$-SMA expression in kidney cortex at all doses tested compared with vehicle (Table 3$)$. Enalapril ( $3 \mathrm{mg} / \mathrm{kg}$ ) modestly reduced $\alpha$-SMA expression in this study, albeit not significantly. Likewise, BI 703704 significantly reduced KIM1 expression at 1,3 , and $10 \mathrm{mg} / \mathrm{kg}$ compared with vehicle and at $10 \mathrm{mg} / \mathrm{kg}$ compared with enalapril $(3 \mathrm{mg} / \mathrm{kg}$ ) (Table 3 ). Enalapril resulted in a modest reduction in KIM-1 expression that was not significantly different from vehicle (Table 3 ).

\section{Discussion}

We investigated the effect of $\mathrm{SGC}$ activation in the ZSF1 rat model of type 2 diabetes mellitus (T2DM)-induced nephropathy. The present study demonstrates that BI 703704, a potent sGC activator, is highly efficacious in preventing the progression of diabetic nephropathy in a preclinical model that displays many of the clinical manifestations of diabetic nephropathy in patients, including obesity, hyperglycemia, dyslipidemia, mild hypertension, proteinuria, and renal lesions. Importantly, ZSF1 rats exhibit markers of oxidative stress, as evidenced by increased levels of urinary 8-hydroxy2-deoxyguanosine (Prabhakar et al., 2007). Given that oxidative stress can result in an inactivation of sGC through oxidation of its heme prosthetic group, it was important to test the effectiveness of a sGC activator in such a setting. Our data demonstrate that BI 703704 is effective in elevating renal cGMP levels in a dose-dependent manner and in slowing the progression of diabetic nephropathy in an oxidative stress milieu. 

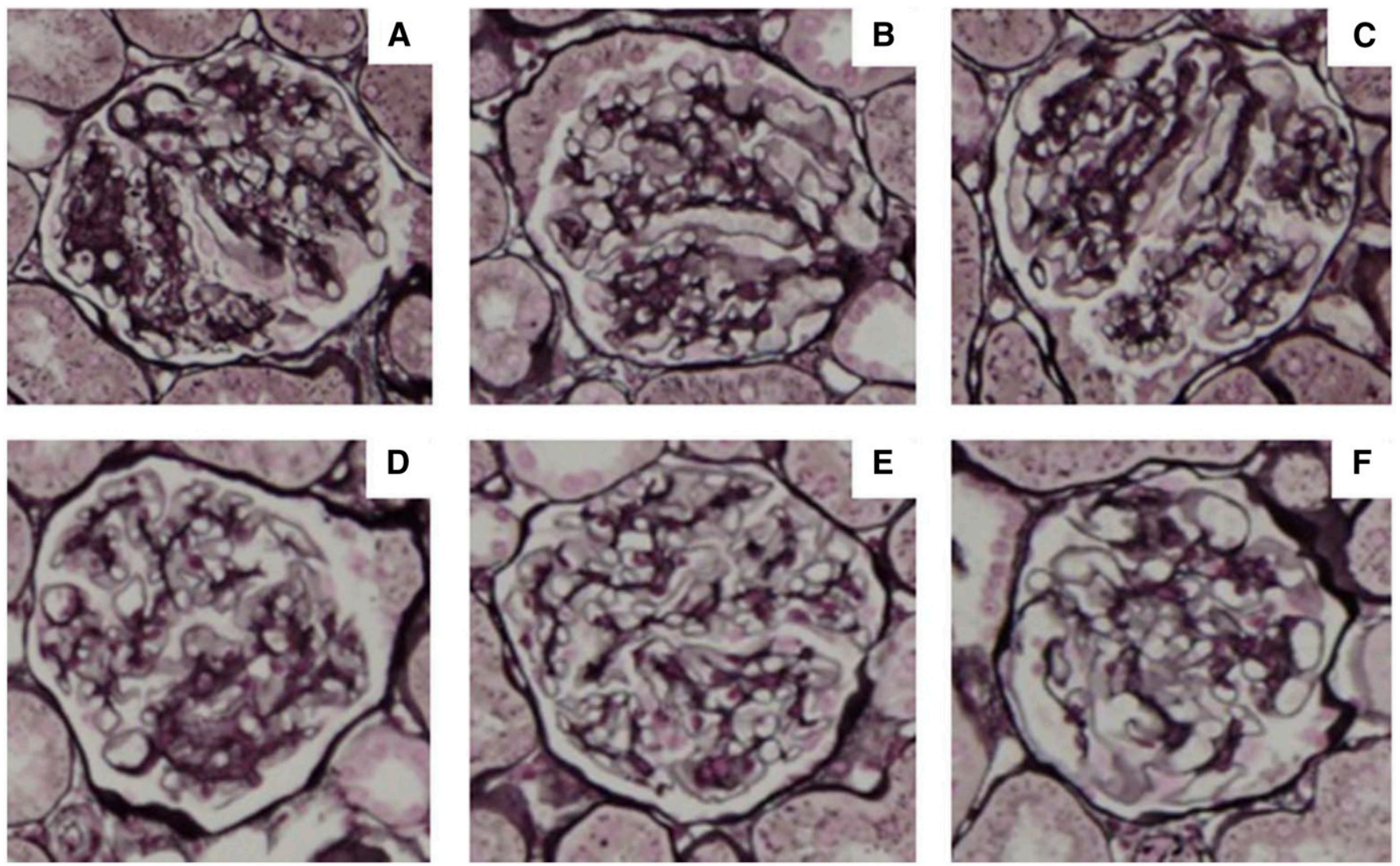

Fig. 7. Effect of sGC activation on glomerular lesions. Representative glomerulosclerosis from (A) vehicle, (B) enalapril at $3 \mathrm{mg} / \mathrm{kg}$, and (C-F) BI 703704 at $0.3,1,3$, and $10 \mathrm{mg} / \mathrm{kg}$, respectively. Glomerular semiquantitative scoring performed with periodic acid methenamine on 25 random glomeruli and scored as follows: $1^{+}$, showing segmental condensation and structural collapse of approximately one quarter of the glomerular area; $2^{+}$, showing segmental condensation and structural collapse of half of the glomerular area; and $3^{+}$, showing condensation and structural collapse of most of the glomerular area. Red arrows indicate areas of advanced glomerulosclerosis in the vehicle control tissue. BI 703704 resulted in a dose-dependent reduction in glomerulosclerosis.

Given that the ZSF1 rat does not exhibit a decline in glomerular filtration rate by 27 weeks of age, proteinuria and renal histologic measures of glomerulosclerosis and tubulointerstitial lesions were assessed as surrogate measures of renal function. Proteinuria has been demonstrated to be a strong risk factor for renal functional decline (de Zeeuw et al., 2004). Furthermore, reductions in proteinuria with ARBs have been shown to be predictive of renal functional outcome in patients with diabetic nephropathy (Brenner et al., 2001; Lewis et al., 2001). The sGC activator BI 703704 resulted in significant, dosedependent reductions in UPE in ZSF1 rats within 15 weeks of treatment. Notably, this was achieved at doses of BI $703704(0.3$, 1 , and $3 \mathrm{mg} / \mathrm{kg}$ ) that did not significantly reduce MAP. This is consistent with a previous report indicating that treatment with the sGC activator HMR1766 can reduce urinary albumin excretion in the rat 5/6 nephrectomy model of chronic renal failure without affecting blood pressure (Benz et al., 2007). Thus, although BI 703704 is capable of reducing blood pressure, significant hemodynamic effects are not required to induce robust proteinuria reductions in ZSF1 rats. Moreover, the potency of BI 703704 to reduce proteinuria was consistent with the potency of the molecule to elevate renal cGMP, thereby demonstrating that these antiproteinuric effects are due to sGC activation. Thus, our results confirm the mechanism of action of BI 703704 in vivo.

A hallmark of diabetic nephropathy is the development of glomerulosclerosis. Severity of glomerular lesions has been shown to be associated with progression to ESRD and doubling of serum creatinine in T2DM patients with biopsy-proven diabetic nephropathy (An et al., 2015). BI 703704 produced a dose-dependent reduction in the incidence of glomerulosclerosis in ZSF1 rats when compared with vehicle. Similar to effects on proteinuria, reductions in glomerulosclerosis were observed at doses of BI 703704 (1 and $3 \mathrm{mg} / \mathrm{kg}$ ) that did not significantly affect blood pressure. These results confirm the efficacy of BI 703704, a sGC activator, in preventing glomerular remodeling, thereby inhibiting disease progression.

Tubulointerstitial remodeling, including interstitial fibrosis and volume, has been shown to correlate with serum creatinine concentrations in diabetic nephropathy patients (Bader et al., 1980). In this study, we assessed tubulointerstitial lesions by measuring the number of foci showing mild to severe lesions indicative of an inflammatory and profibrotic milieu. Treatment with BI 703704 dose-dependently reduced the number of interstitial foci, with a significant effect observed at the highest dose compared with vehicle. This was accompanied with a decrease in $\alpha$-SMA staining, a marker of myofibroblast activation, thereby supporting an antifibrotic effect of BI 703704. Furthermore, BI 703704 effectiveness in preventing tubulointerstitial damage was supported by significant reductions in KIM-1 staining, a marker of tubular injury. Taken together, these results demonstrated the efficacy of a sGC activator in preventing tubulointerstitial damage in a model of T2DM-induced nephropathy. 

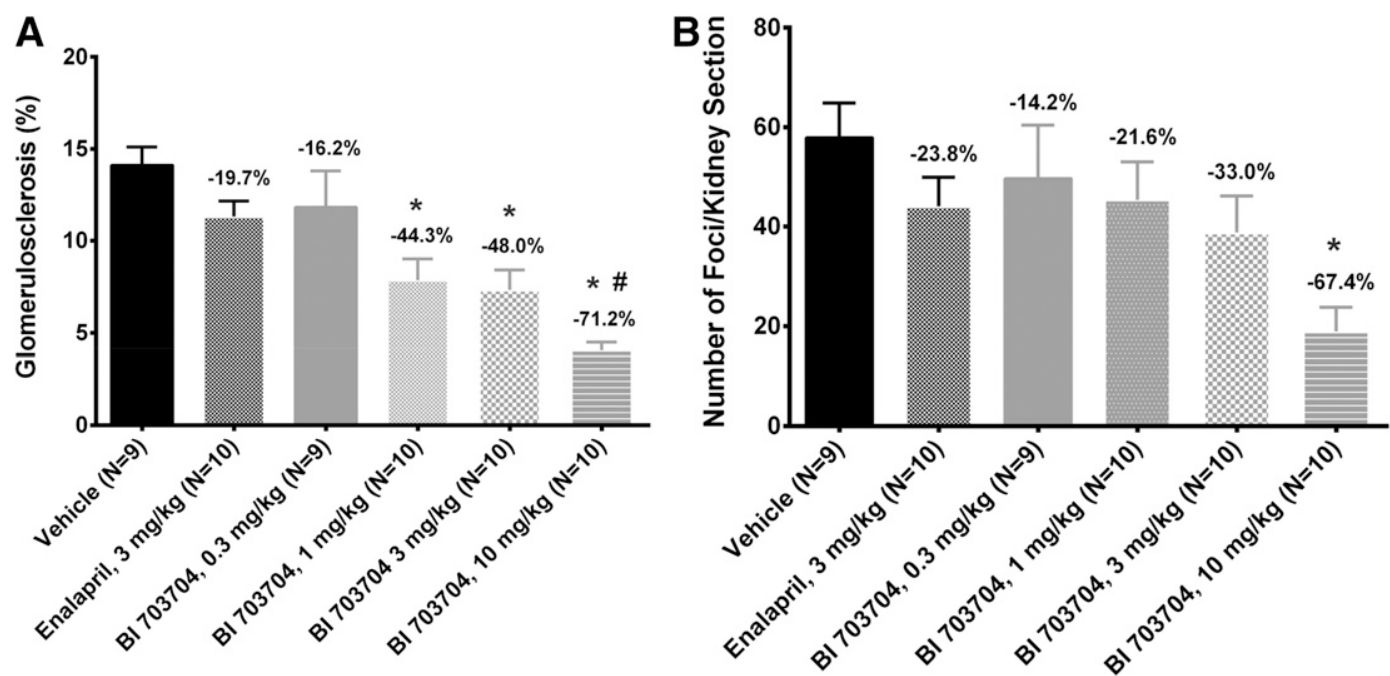

Fig. 8. Effect of sGC activation on incidence of glomerulosclerosis (A) and tubulointerstitial lesions (B). BI 703704 at 1,3 , and $10 \mathrm{mg} / \mathrm{kg}$ dosedependently and significantly reduced the incidence of glomerulosclerosis (A, glomerulosclerosis \%) and interstitial lesions (B, the number of foci/kidney section). ${ }^{* \#}$ Signifies statistical significance compared with vehicle and enalapril $(3 \mathrm{mg} / \mathrm{kg})$, respectively $(P<0.05$, one-way analysis of variance with Tukey's post hoc analysis).

Currently, approved therapies for the treatment of diabetic nephropathy consist of ACE inhibitors and ARBs (Lewis et al., 1993, 2001; Brenner et al., 2001). In our study, enalapril, used as a positive control, significantly reduced proteinuria, but failed to significantly prevent glomerulosclerosis and interstitial lesions. Importantly, the magnitude of enalapril antihypertensive and antiproteinuric effects was consistent with that seen in clinical trials (The SOLVD Investigators, 1991; Brenner et al., 2001; Lewis et al., 2001), indicative that the dose selected ( $3 \mathrm{mg} / \mathrm{kg})$ was clinically relevant. It is noteworthy that BI 703704 produced similar or lesser blood pressure reductions than enalapril in our study at all but the highest dose, yet provided greater renal protection, as evident by significant reductions in glomerular and interstitial lesions. This suggests that sGC activation provides renal protection independent of blood pressure effects, and that ACE inhibitors and SGC activators may affect different molecular pathways.

The role of the sGC-cGMP pathway in metabolic regulation is emerging. A hyperinsulinemic euglycemic clamp performed in high-fat diet-fed mice indicated that treatment with a PDE5 inhibitor and NO donor improved significantly insulin sensitivity compared with control (Ayala et al., 2007). In addition, recent studies demonstrate protection from dietinduced obesity with a sGC stimulator. This effect was accompanied with browning of fat mass, improved glucose tolerance, increased food intake, and increased energy expenditure (Hoffmann et al., 2015). Consistent with these beneficial effects on glycemic control, our data indicate a reduction in $\mathrm{HbA} 1 \mathrm{c}$ at the doses of 3 and $10 \mathrm{mg} / \mathrm{kg}$ BI 703704. Importantly, these findings cannot be solely attributed to increased cGMP, as HbA1c lowering occurred in this study concomitantly with a reduction in food intake, an effect that may be resulting from taste aversion. Despite the reduction in food intake, BI 703704-treated rats exhibited an increase in body weight that may be driven by a reduction in energy expenditure. Nonetheless, the beneficial effects on glycemia raise the possibility that renal protection may in part be the indirect result of such an improvement. However, given that significant effects on proteinuria and renal histologic measures were observed at the dose of $1 \mathrm{mg} / \mathrm{kg}$, a dose that did not significantly affect $\mathrm{HbA} 1 \mathrm{c}$, our data are also supportive of glucose-independent effects of sGC activation on the kidney. Moreover, as the ZSF1 rat is leptin receptor deficient, the metabolic effects BI 703704 on body weight and food intake cannot be adequately interpreted in this model. Future studies will further investigate these effects, utilizing suitable animal models for such measures such as diet-induced obesity rodents.

Few published reports have explored the utility of sGC activators for the treatment of chronic kidney disease. Both BAY 58-2667 and HMR1766 provided renal protection in the

TABLE 3

Effect of sGC activation on markers of renal damage

\begin{tabular}{|c|c|c|c|c|}
\hline \multirow{2}{*}{ Treatment } & \multicolumn{2}{|c|}{$\alpha$-SMA } & \multicolumn{2}{|c|}{ KIM-1 } \\
\hline & $\%$ Positive Expression & Percent Change & $\%$ Positive Expression & Percent Change \\
\hline Vehicle & $6.08 \pm 0.70$ & & $3.15 \pm 0.45$ & \\
\hline Enalapril $3 \mathrm{mg} / \mathrm{kg}$ & $4.45 \pm 0.49$ & -26.7 & $2.28 \pm 0.31$ & -27.5 \\
\hline BI $7037040.3 \mathrm{mg} / \mathrm{kg}$ & $2.09 \pm 0.36$ & $-65.6^{* \#}$ & $1.94 \pm 0.22$ & -38.4 \\
\hline $\mathrm{BI} 7037041 \mathrm{mg} / \mathrm{kg}$ & $1.65 \pm 0.39$ & $-72.9 * \#$ & $1.29 \pm 0.26$ & $-59.0 *$ \\
\hline BI $7037043 \mathrm{mg} / \mathrm{kg}$ & $1.92 \pm 0.47$ & $-68.4^{* \#}$ & $1.54 \pm 0.30$ & $-51.2^{*}$ \\
\hline $\mathrm{BI} 70370410 \mathrm{mg} / \mathrm{kg}$ & $1.97 \pm 0.30$ & $-67.6^{* \#}$ & $0.89 \pm 0.20$ & $-71.8^{* \#}$ \\
\hline
\end{tabular}

Markers of renal damage, $\alpha$-SMA and KIM-1, were quantified by immunohistochemistry and expressed as mean \pm S.E.M. percent positive expression area/glomeruli and as a percent change from vehicle control.

${ }^{*}$ "\# Significant $P<0.05$ versus vehicle and enalapril, respectively, using one-way analysis of variance. 
rat 5/6 nephrectomy model of chronic renal failure (Kalk et al., 2006; Benz et al., 2007). This model is characterized by surgically-induced impaired renal function, but lacks the diabetic milieu and its impact on renal molecular pathways. BAY 58-2667 also reduced serum creatinine, blood urea nitrogen levels, and improved survival in hypertensive transgenic renin rats [TG(mRen2)27 rats] treated with a nitric oxide inhibitor, supporting the persistent efficacy of a sGC activator in the presence of oxidative stress (Stasch et al., 2006). Additionally, sGC stimulators have been investigated for their effects on renal function in models of kidney disease (Stasch et al., 2015). Notably, the effect of the sGC stimulator riociguat was tested alone and in combination with telmisartan, an ARB, in the streptozotocin-treated eNOS-deficient mouse, a model of type 1 diabetes and endothelial dysfunction (Ott et al., 2012). Although monotherapy with riociguat produced a significant decrease $(\sim 9 \mathrm{mmHg})$ in systolic blood pressure, there was no effect on albuminuria or glomerulosclerosis with 11 weeks of treatment, possibly due to sGC heme oxidation, which renders the enzyme nonresponsive to stimulators. In contrast, BI 703704 significantly reduced proteinuria in the ZSF1 rat with no notable effects on blood pressure, supporting a greater efficacy of sGC activators compared with stimulators in the presence of oxidative stress. Future studies directly comparing sGC activators and stimulators in diabetes-induced nephropathy models will provide further insights into the potential utility of these two modalities for treating diabetic nephropathy. Furthermore, studies aimed at investigating the renoprotective effects of sGC activators when combined with the standard of care (ACE inhibitors, ARBs) are warranted.

To our knowledge, this is the first report of renal beneficial effects, including histologic improvements, of a sGC activator in a model of type 2 diabetes-induced nephropathy. Importantly, we demonstrate beneficial effects of BI 703704 in the absence of significant hemodynamic effects. Therefore, we propose sGC activation as a novel pharmacological approach for the treatment of diabetic nephropathy.

\section{Authorship Contributions}

Participated in research design: Boustany-Kari, Harrison, Chen, Lincoln, Qian, Clifford, Wong, Gueneva-Boucheva, Fryer, Richman, Sarko, Pullen.

Conducted experiments: Harrison, Chen, Lincoln, Clifford, Wang, Zhang.

Contributed new reagents or analytic tools: Bosanac, Sarko.

Performed data analysis: Chen, Wang, Wong, Gueneva-Boucheva.

Wrote or contributed to the writing of the manuscript: BoustanyKari, Harrison, Qian, Wong, Gueneva-Boucheva, Fryer, Sarko, Pullen.

\section{References}

An Y, Xu F, Le W, Ge Y, Zhou M, Chen H, Zeng C, Zhang H, and Liu Z (2015) Renal histologic changes and the outcome in patients with diabetic nephropathy. Nephrol Dial Transplant 30:257-266.

Ayala JE, Bracy DP, Julien BM, Rottman JN, Fueger PT, and Wasserman DH (2007) Chronic treatment with sildenafil improves energy balance and insulin action in high fat-fed conscious mice. Diabetes 56:1025-1033.

Bader R, Bader H, Grund KE, Mackensen-Haen S, Christ H, and Bohle A (1980) Structure and function of the kidney in diabetic glomerulosclerosis: correlations between morphological and functional parameters. Pathol Res Pract 167:204-216. Benz K, Orth SR, Simonaviciene A, Linz W, Schindler U, Rütten H, and Amann K (2007) Blood pressure-independent effect of long-term treatment with the soluble heme-independent guanylyl cyclase activator HMR1766 on progression in a mode of noninflammatory chronic renal damage. Kidney Blood Press Res 30:224-233.

Berry A, Bosanac T, Ginn JD, Hopkins TD, Schlyer S, Soleymanzadeh F, Westbrook J, Yu M, and Zhang Z (2013) Soluble Guanylate Cyclase Activators U.S. patent WO 2013/0203729 A1 2013 Feb 21.
Boerrigter G and Burnett JC, Jr (2007) Nitric oxide-independent stimulation of soluble guanylate cyclase with BAY 41-2272 in cardiovascular disease. Cardiovasc Drug Rev 25:30-45.

Brenner BM, Cooper ME, de Zeeuw D, Keane WF, Mitch WE, Parving H-H, Remuzzi G, Snapinn SM, Zhang Z, and Shahinfar S; RENAAL Study Investigators (2001) Effects of losartan on renal and cardiovascular outcomes in patients with type 2 diabetes and nephropathy. $N$ Engl J Med 345:861-869.

Dellamea BS, Ferreira Pinto LC, Leitao CB, Santos KG, and Santos Canani LH (2014) Endothelial nitric oxide synthase gene polymorphisms and risk of diabetic nephropathy: a systemic review and meta-analysis. BMC Med Genet 15:9:1-13.

de Zeeuw D, Remuzzi G, Parving H-H, Keane WF, Zhang Z, Shahinfar S, Snapinn S, Cooper ME, Mitch WE, and Brenner BM (2004) Proteinuria, a target for renoprotection in patients with type 2 diabetic nephropathy: lessons from RENAAL. Kidney Int 65:2309-2320.

Hanai K, Babazono T, Nyumura I, Toya K, Tanaka N, Tanaka M, Ishii A and Iwamoto Y (2009) Asymmetric dimethylarginine is closely associated with the development and progression of nephropathy in patients with type 2 diabetes. Nephrol Dial Transplant 24:1884-1888.

Hodgin JB, Nair V, Zhang H, Randolph A, Harris RC, Nelson RG, Weil EJ, Cavalcoli JD, Patel JM, and Brosius FC, 3rd, et al. (2013) Identification of cross-species shared transcriptional networks of diabetic nephropathy in human and mouse glomeruli. Diabetes 62:299-308.

Hoffmann LS, Etzrodt J, Willkomm L, Sanyal A, Scheja L, Fischer AW, Stasch JP, Bloch W, Friebe A, Heeren J, and Pfeifer A (2015).Stimulation of soluble guanylyl cyclase protects against obesity by recruiting brown adipose tissue. Nat Commun 6 : 7235

Kalk P, Godes M, Relle K, Rothkegel C, Hucke A, Stasch J-P, and Hocher B (2006) NO-independent activation of soluble guanylate cyclase prevents disease progression in rats with 5/6 nephrectomy. Br J Pharmacol 148:853-859.

Lajer M, Tarnow L, Jorsal A, Teerlink T, Parving H-H, and Rossing P (2008) Plasma concentration of asymmetric dimethylarginine (ADMA) predicts cardiovascular morbidity and mortality in type 1 diabetic patients with diabetic nephropathy. Diabetes Care 31:747-752.

Lewicki JA, Brandwein HJ, Mittal CK, Arnold WP, and Murad F (1982) Properties of purified soluble guanylate cyclase activated by nitric oxide and sodium nitroprusside. J Cyclic Nucleotide Res 8:17-25.

Lewis EJ, Hunsicker LG, Bain RP, and Rohde RD; The Collaborative Study Group (1993) The effect of angiotensin-converting-enzyme inhibition on diabetic nephropathy. N Engl J Med 329:1456-1462.

Lewis EJ, Hunsicker LG, Clarke WR, Berl T, Pohl MA, Lewis JB, Ritz E, Atkins RC, Rohde R, and Raz I; Collaborative Study Group (2001) Renoprotective effect of the angiotensin-receptor antagonist irbesartan in patients with nephropathy due to type 2 diabetes. $N$ Engl $J$ Med 345:851-860.

Ott IM, Alter ML, von Websky K, Kretschmer A, Tsuprykov O, Sharkovska Y, Krause-Relle K, Raila J, Henze A, Stasch J-P, and Hocher B (2012) Effects of stimulation of soluble guanylate cyclase on diabetic nephropathy in diabetic eNOS knockout mice on top of angiotensin II receptor blockade. PLoS One 7:e42623.

Persson F, Rossing P, Hovind P, Stehouwer CDA, Schalkwijk CG, Tarnow L, and Parving $\mathrm{H}-\mathrm{H}$ (2008) Endothelial dysfunction and inflammation predict development of diabetic nephropathy in the Irbesartan in Patients with Type 2 Diabetes and Microalbuminuria (IRMA 2) study. Scand J Clin Lab Invest 68:731-738.

Prabhakar S, Starnes J, Shi S, Lonis B, and Tran R (2007) Diabetic nephropathy is associated with oxidative stress and decreased renal nitric oxide production. J Am Soc Nephrol 18:2945-2952.

Saran R, Li Y, Robinson B, Ayanian J, Balkrishnan R, Bragg-Gresham J, Chen JTL, Cope E, Gipson D, He K, et al. (2015) US Renal Data System 2014 Annual Data Report: Epidemiology of Kidney Disease in the United States. Am J Kidney Disease 66 (Suppl 1):Svii, S1-S305.

Shibata R, Ueda S, Yamagishi S, Kaida Y, Matsumoto Y, Fukami K, Hayashida A, Matsuoka H, Kato S, and Kimoto M, et al. (2009) Involvement of asymmetric dimethylarginine (ADMA) in tubulointerstitial ischaemia in the early phase of diabetic nephropathy. Nephrol Dial Transplant 24:1162-1169.

Stasch J-P, Becker EM, Alonso-Alija C, Apeler H, Dembowsky K, Feurer A, Gerzer R, Minuth T, Perzborn E, and Pleiss U, et al. (2001) NO-independent regulatory site on soluble guanylate cyclase. Nature 410:212-215.

Stasch J-P, Dembowsky K, Perzborn E, Stahl E, and Schramm M (2002) Cardiovascular actions of a novel NO-independent guanylyl cyclase stimulator, BAY 418543: in vivo studies. $B r$ J Pharmacol 135:344-355.

Stasch J-P, Schlossmann J, and Hocher B (2015) Renal effects of soluble guanylate cyclase stimulators and activators: a review of the preclinical evidence. Curr Opin Pharmacol 21:95-104.

Stasch J-P, Schmidt PM, Nedvetsky PI, Nedvetskaya TY, H S AK, Meurer S, Deile M, Taye A, Knorr A, and Lapp H, et al. (2006) Targeting the heme-oxidized nitric oxide receptor for selective vasodilatation of diseased blood vessels. J Clin Invest 116 : 2552-2561.

Tervaert TW, Mooyaart AL, Amann K, Cohen AH, Cook HT, Drachenberg CB, Ferrario F, Fogo AB, Haas M, and de Heer E, et al.; Renal Pathology Society (2010) Pathologic classification of diabetic nephropathy. J Am Soc Nephrol 21:556-563.

The SOLVD Investigators (1991) Effect of enalapril on survival in patients with reduced left ventricular ejection fractions and congestive heart failure. $N \mathrm{Engl} J$ Med 325:293-302.

Tofovic SP and Jackson EK (2003) Rat Models of the Metabolic Syndrome in Methods in Molecular Medicine 86, Renal Disease: Techniques and Protocols (Goligorsky MS ed) pp 29-36, Humana Press, Tolowa, NJ.

Address correspondence to: Dr. Carine M. Boustany-Kari, 900 Ridgebury Road, PO Box 368, Ridgefield, CT 06877. E-mail: carine.boustany@boehringeringelheim.com 